\title{
Negative and positive dust grain effect on the modulation instability of an intense laser propagating in a hot magnetoplasma
}

\author{
N. Sepehri Javan ${ }^{1}$
}

Received: 20 March 2017/Accepted: 31 July 2017/Published online: 7 August 2017

(C) The Author(s) 2017. This article is an open access publication

\begin{abstract}
The modulation instability of intense circularly polarized laser beam in hot magnetized dusty plasma is studied. A nonlinear equation describing the interaction of laser with dusty plasma in the quasi-neutral approximation is derived. The effect of negative and positive dust grains on the laser modulation growth rate is studied. It is shown that the existence of positive dust grains instead of ions can substantially improve the modulation growth rate.
\end{abstract}

Keywords Dusty plasma - Laser · Modulation · Nonlinear ineration - Growth rate $\cdot$ Magnetoactive

\section{Introduction}

Dusty plasmas are frequently found in different places of the cosmic environment. They exist in planetary rings, comet comae and tails, and interplanetary and interstellar molecular clouds [1-7]. They can be revealed in the vicinity of aircrafts $[6,7]$ and in the controlled plasma fusion [8-10]. In some industrial applications of plasma such as plasma processing of materials, the formation of dusty plasma has also been observed [11-14]. They can also be created during laser ablation experiments $[15,16]$. In addition, complex dusty plasmas form in the flame of a humble candle, in the zodiacal light, cloud-to-ground lightings, and volcanic eruptions. Recently [17], it has been suggested that the ball lightning is the dusty plasma medium and it is created during oxidation of nanoparticle networks in the normal

N. Sepehri Javan

sepehri_javan@uma.ac.ir

1 Department of Physics, University of Mohaghegh Ardabili, PO Box 179, Ardabil, Iran lightning strike on soil. Furthermore, dusty plasmas can be produced and investigated in laboratories. Dust grains not only can be intentionally added into the plasma, but can also appear because of different mechanisms in some experiments. The existence of heavy and highly ionized dust grains gives some special and extraordinary properties to the dusty plasma, providing great motivations to investigate it theoretically and experimentally. One of these interests is the study of interaction of laser with dusty plasmas and its related linear and nonlinear effects. These effects include wave dissipation [18], modulation and filamentation instabilities [19-22], linear and nonlinear wave propagation [18, 23-31], parametric instabilities [32], self-focusing [18, 33], etc. Moreover, interaction of laser with dusty plasmas has some important industrial applications. For instance, by interaction of high power lasers with molecular or atomic clusters, during which dusty plasma is created, high-energy electrons can be produced by three processes, i.e., inner ionization, outer ionization, and Coulomb explosion [34-36]. In some experiments, lasers are used in order to study the dynamics of different phenomena in dusty plasmas which some recent experiments about investigation of different exotic phenomena can be found in [37-42].

Here, we focus on the MI of intense lasers in magnetized dusty plasmas. The MI represents a fundamental subject in the theory of nonlinear waves. MI exists due to the interplay between the nonlinearity and dispersion/diffraction effects. The ponderomotive force created by the electromagnetic wave (EMW) stimulates low-frequency perturbations of the electrons density; then, they interact with the primary highfrequency EMW in which the amplitude of the pump wave becomes modulated and the MI of the EMW occurs. The MI of laser beams in plasmas and dielectrics has been the subject of several publications [43-45]. The MI of strong EMWs in plasmas with arbitrary large amplitude was 
studied by Shukla et al. in 1987 [46]. Most of the early publications about MI considered one-dimensional models in which the laser beam was represented as a plane wave $[47,48]$. The MI of a laser pulse in the cold nonmagnetized plasma has been considered by several authors $[46,49,50]$. The MI of a linearly polarized laser pulse propagating in the cold magnetized plasma was studied by Jha et al. in 2005 [51]. The MI of the right-hand elliptically laser pulse in cold magnetized plasma has been investigated by Chen et al. in 2011 [52]. Recently, the MI of an intense circularly polarized laser beam in the hot magnetized electron-positron and electron-ion (e-i) plasmas as studied by Sepehri Javan $[53,54]$. Our recent work [55] has extended the MI of the circularly polarized laser beam propagating along an external magnetic field in the non-Maxwellian plasma. In this article we study the MI of an intense laser beam in the magnetized hot dusty plasma. In the quasi-neutral approximation and by using a relativistic fluid model, we consider the presence of both negative and positive dust grains and investigate the effect of such grains on the MI. The organization of the paper is as follows. In Sect. 2, the basic assumptions are presented and a nonlinear wave equation is derived for the laser amplitude evolutions. An analytic expression for the growth rate of MI is obtained in Sect. 3. In Sect. 4, a numerical study of the MI of circularly polarized laser beam in the magnetized electron-ion-positive dustnegative dust $(\mathrm{e}-\mathrm{i}-\mathrm{d}+-\mathrm{d}-)$ plasma is presented. The concluding remarks are made in Sect. 5.

\section{Deriving a nonlinear wave equation}

Let us consider the propagation of a circularly polarized EMW in a hot magnetized four-component plasma which contains electron, ion, and positive and negative dust grains. Each type of plasma particle may have its own specific temperature. To determine the quantities related to the electrons, ions, and positive and negative dust grains, we use indices e, $\mathrm{i}, \mathrm{d}+$ and $\mathrm{d}-$, respectively. We take the external magnetic field parallel to the $z$ axis, i.e., $\mathbf{B}_{\mathbf{0}}=B_{0} \hat{\mathbf{e}}_{\mathbf{z}}$. To describe the nonlinear dynamics of the interaction of EMW with the dusty plasma, we define the electric and magnetic fields $\mathbf{E}$ and $\mathbf{B}$ through the vector and scalar potentials $\mathbf{A}, \varphi$ as:

$\mathbf{E}=-\frac{1}{c} \frac{\partial \mathbf{A}}{\partial t}-\nabla \varphi, \quad \mathbf{B}=\nabla \times \mathbf{A}$,

where $c$ is the speed of light.

Using Eq. (1) in Maxwell equations, one can easily obtain:

$\frac{1}{c^{2}} \frac{\partial^{2} \mathbf{A}}{\partial t^{2}}-\nabla^{2} \mathbf{A}=\frac{4 \pi}{c} \mathbf{J}$, where $\mathbf{J}=-n_{e} e \mathbf{v}_{e}$ is the current density of electrons, $e, \mathbf{v}_{e}$ and $n_{e}$ are the density, velocity and charge of the electron, respectively. We ignore the translational velocity of the heavy ions and dust grains. Now, we can write the relativistic fluid momentum equation for electrons as:

$\frac{\partial \mathbf{p}_{e}}{\partial t}+\left(\mathbf{v}_{e} . \nabla\right) \mathbf{p}_{e}=-e\left[\mathbf{E}+\frac{1}{c} \mathbf{v}_{e} \times\left(\mathbf{B}+\mathbf{B}_{\mathbf{0}}\right)\right]-\frac{1}{n_{e}} \nabla \Pi_{e}$,

where $\mathbf{p}_{e}$ and $\Pi_{e}$ are the momentum and pressure of the electron, respectively. Substituting Eq. (1) into Eq. (3) leads to

$$
\begin{aligned}
& \frac{\partial \mathbf{p}_{e}}{\partial t}+\frac{1}{\gamma_{e} m_{0}}\left(\mathbf{p}_{e} \cdot \nabla\right) \mathbf{p}_{e}=\frac{e}{c} \frac{\partial \mathbf{A}}{\partial t}+e \nabla \varphi-\frac{e}{\gamma_{e} m_{0} c} \mathbf{p}_{e} \\
& \times(\nabla \times \mathbf{A})-\frac{\omega_{c}}{\gamma_{e}} \mathbf{p}_{e} \times \hat{\mathbf{e}}_{\mathbf{z}}-k_{B} T_{e} \nabla \ln \left(n_{e}\right),
\end{aligned}
$$

where $T_{e}$ is the temperature of electrons, $m_{0}$ the electron rest mass, $\gamma_{e}=\sqrt{1+p_{e}^{2} / m_{0}^{2} c^{2}}$ the relativistic Lorentz factor, $\omega_{c}=e B_{0} / m_{0} c$ the electron cyclotron frequency and $k_{\mathrm{B}}$ the Boltzmann constant.

We consider the propagation of circularly polarized wave along the external magnetic field and write the vector potential of this wave as:

$\mathbf{A}=\frac{1}{2} \tilde{A}\left(\hat{\mathbf{e}}_{\mathbf{x}}+i \sigma \hat{\mathbf{e}}_{y}\right) \exp \left(-i \omega_{0} t+i k_{0} z\right)+c . c .$,

where $\omega_{0}, k_{0}$ are the frequency and wave number, respectively. $\sigma=+1,-1$ denotes the right- and left-hand circularly polarized wave, respectively, and also $\tilde{A}(z, t)$ is the slowly varying amplitude that satisfies the following condition:

$\left|\frac{1}{\omega_{0}} \frac{\partial \tilde{A}}{\partial t}\right|<<|\tilde{A}|$.

Inserting Eq. (5) into Eq. (4), we can find that Eq. (4) is satisfied by [56-58]:

$\overline{\mathbf{p}}_{e}=\frac{\overline{\mathbf{A}}}{1-\sigma \alpha / \gamma_{e}}$,

and together with

$n_{e}=n_{0 e} \exp \left[\frac{e \varphi}{k_{B} T_{e}}-\beta_{e}\left(\gamma_{e}-1-\frac{\sigma \alpha}{2 \gamma_{e}^{2}}\left|\bar{p}_{e}\right|^{2}\right)\right]$,

where $n_{0 e}$ is the unperturbed density of electrons, $\overline{\mathbf{p}}_{e}=$ $\mathbf{p}_{e} / m_{0} c$ is the normalized electron momentum, $\overline{\mathbf{A}}=$ $e \mathbf{A} / m_{0} c^{2}$ is the normalized vector potential, $\alpha=\omega_{c} / \omega_{0}$, $\beta_{e}=c^{2} / v_{T_{e}}^{2}$ and $v_{T_{e}}^{2}=k_{\mathrm{B}} T_{e} / m_{0}$ is the electron thermal velocity. For weakly relativistic laser intensity, when $|\overline{\mathbf{A}}|^{2},\left|\overline{\mathbf{P}}_{e}\right|^{2}<<1$ and $\gamma_{e} \approx 1+\frac{1}{2}\left|\overline{\mathbf{P}}_{e}\right|^{2}$, we can simplify density of electrons as follows: 
$n_{e}=n_{0 e} \exp \left[\frac{e \varphi}{k_{B} T_{e}}-\frac{\beta_{e}}{2} \frac{|\overline{\mathbf{A}}|^{2}}{(1-\sigma \alpha)}\right]$.

We suppose that the ion and dust grains slow motions are non-relativistic and, by assuming an isothermal equation of state for these heavy particles, obtain the following expressions for number densities:

$n_{i}=n_{0 i} \exp \left(-\frac{e \varphi}{k_{\mathrm{B}} T_{i}}\right)$,

$n_{\mathrm{d}+}=n_{0 \mathrm{~d}+} \exp \left(-\frac{z_{+} e \varphi}{k_{\mathrm{B}} T_{\mathrm{d}+}}\right)$,

$n_{\mathrm{d}-}=n_{0 \mathrm{~d}-} \exp \left(\frac{z_{-} e \varphi}{k_{\mathrm{B}} T_{\mathrm{d}-}}\right)$,

where $T_{j}, n_{0 j}, z_{+}$and $z_{-}$are the temperature and unperturbed density of $j$-type particle, and order of ionization of positive and negative dust grains, respectively.

Expanding Eqs. (9)-(12) and using them in the quasineutral condition, i.e., $n_{i}+z_{+} n_{\mathrm{d}+}-n_{e}-z_{-} n_{\mathrm{d}-}=0$, yield the following result:

$\Phi=\frac{e \varphi}{k_{\mathrm{B}} T_{e}}=\frac{\mu \beta_{e}|\overline{\mathbf{A}}|^{2}}{2(1-\sigma \alpha)}$,

where

$\mu=\frac{1}{1+\zeta_{i} \delta_{i}^{-1}+z_{+}^{2} \zeta_{\mathrm{d}+} \delta_{\mathrm{d}+}^{-1}+z_{-}^{2} \zeta_{\mathrm{d}-} \delta_{\mathrm{d}-}^{-1}}$,

$\zeta_{j}=\frac{n_{0 j}}{n_{0 e}}, \quad \delta_{j}=\frac{T_{j}}{T_{e}}, \quad j=i, \mathrm{~d}+, \mathrm{d}-$.

Substituting Eq. (13) into Eq. (9) results in the following expression for the electron density:

$n_{e}=n_{0 e} \exp \left[\frac{(\mu-1) \beta_{e}}{2(1-\sigma \alpha)}|\overline{\mathbf{A}}|^{2}\right]$.

In physical units, from Eq. (7) we can obtain the following for the velocity of electrons:

$\mathbf{v}_{e}=\frac{e}{m_{0 e} c} \frac{\mathbf{A}}{\gamma_{e}-\sigma \alpha}$.

Also, for electron Lorentz factor, we can approximately write:

$\gamma_{e} \approx \sqrt{1+\frac{|\overline{\mathbf{A}}|^{2}}{(1-\sigma \alpha)^{2}}}$.

Now, taking Eqs. (16) and (17) into consideration, we derive the nonlinear current density as follows:

$-\frac{4 \pi}{c} \mathbf{J}=\frac{\omega_{p}^{2}}{c^{2}} \frac{\mathbf{A}}{\left(\gamma_{e}-\sigma \alpha\right)} \exp \left[\frac{(\mu-1) \beta_{e}}{2(1-\sigma \alpha)}|\overline{\mathbf{A}}|^{2}\right]$,

where $\omega_{p}=\sqrt{4 \pi n_{0} e^{2} / m_{0}}$ is the electron Langmuir frequency.
In the weakly relativistic regime of laser intensity, we can expand the nonlinear current density of Eq. (19) with respect to the normalized vector potential amplitude and save only the second orders of amplitude. In this case, substituting simplified current density, together with the vector potential in the form of Eq. (5) into Eq. (2) leads to the following equation for the EMW envelope evolutions:

$$
\left(\nabla^{2}-\frac{1}{c^{2}} \frac{\partial^{2}}{\partial t^{2}}\right) a e^{i\left(k_{0} z-\omega_{0} t\right)}=k_{p}^{2}\left[\frac{1}{1-\sigma \alpha}-|a|^{2} N\right] a e^{i\left(k_{0} z-\omega_{0} t\right)},
$$

where

$N=\frac{\omega_{0}^{4}}{2\left(\omega_{0}-\sigma \omega_{c}\right)^{4}}+\frac{\omega_{0}^{2}}{2\left(\omega_{0}-\sigma \omega_{c}\right)^{2}}(1-\mu) \beta_{e}$,

and $a=e \tilde{A} / m_{0} c^{2}, k_{p}=\omega_{p} / c$ are the normalized amplitude of vector potential, and wave number of the plasma wave, respectively.

For e-i plasma when $\zeta_{i}=1$ and $\zeta_{\mathrm{d}+}=\zeta_{\mathrm{d}-}=0$, the nonlinear term reduces to

$N=\frac{\omega_{0}^{4}}{2\left(\omega_{0}-\sigma \omega_{c}\right)^{4}}+\frac{\omega_{0}^{2}}{2\left(\omega_{0}-\sigma \omega_{c}\right)^{2}} \frac{\beta_{e}}{1+T_{e} / T_{i}}$,

which agrees with the results of Sepehri Javan and Nasirzadeh [56].

\section{Derivation of nonlinear dispersion relation and MI}

To derive the nonlinear dispersion relation, Eq. (18) is simplified in the new form:

$$
\begin{aligned}
& \frac{\partial^{2} a}{\partial t^{2}}-c^{2} \frac{\partial^{2} a}{\partial z^{2}}-2 i \omega_{0} \frac{\partial a}{\partial t}-2 i k_{0} c^{2} \frac{\partial a}{\partial z} \\
& \quad+\left[-\omega_{0}^{2}+c^{2} k_{0}^{2}+\omega_{p}^{2}\left(\frac{1}{1-\sigma \alpha}-N|a|^{2}\right)\right] a \\
& \quad=0 .
\end{aligned}
$$

In the last term of Eq. (23), the coefficient of $a$ is the nonlinear dispersion relation. In the absence of interaction between EMW and plasma, when amplitude is a real constant $\left(a=a_{0}\right)$, we can derive the nonlinear dispersion relation for magnetoplasma with negative and positive dust grains as follows:

$$
\begin{aligned}
& c^{2} k_{0}^{2}-\omega_{0}^{2} \\
& +\omega_{p}^{2}\left[\frac{1}{1-\sigma \alpha}-a_{0}^{2}\left(\frac{\omega_{0}^{4}}{2\left(\omega_{0}-\sigma \omega_{c}\right)^{4}}+\frac{\omega_{0}^{2}}{2\left(\omega_{0}-\sigma \omega_{c}\right)^{2}} \frac{(\mu-1)}{(1-\sigma \alpha)} \beta_{e}\right)\right]=0 .
\end{aligned}
$$

In the linear limit (when $a^{2} \rightarrow 0$ ), Eq. (24) can be reduced to the well-known linear dispersion relation of circularly polarized EMW in the magnetized plasma: 
$k_{0}=\frac{\omega_{0}}{c}\left(1-\frac{\omega_{p}^{2}}{\omega_{0}\left(\omega_{0}-\sigma \omega_{c}\right)}\right)^{1 / 2}$.

It is worth mentioning that in the linear approximation, there is no contribution for dust grains on the dispersion Eq. (25) because we have investigated the evolution of high-frequency EMWs where heavy ions and dust particles cannot respond to this high frequency. However, traces of dust particles can be found in the nonlinear dispersion Eq. (24) through bipolar diffusion caused by slow motion of particles under the influence of ponderomotive laser force and thermal collision force.

By considering the condition of slowly varying amplitude (Eq. 6) and assuming that $\omega_{0}$ and $k_{0}$ satisfy the linear dispersion of Eqs. (23), (25) can be modified as:

$i\left(\frac{\partial a}{\partial t}+v_{g} \frac{\partial a}{\partial z}\right)+\frac{c^{2}}{2 \omega_{0}} \frac{\partial^{2} a}{\partial z^{2}}+\frac{\omega_{p}^{2}}{2 \omega_{0}} N|a|^{2} \quad a=0$

where $v_{g}=\frac{k_{0} c^{2}}{\omega_{0}}$ is the group velocity. Using the following dimensionless variables $\quad \tau=\frac{\omega_{p}^{2}}{\omega_{0}} t, \quad U_{g}=\frac{\omega_{0}}{\omega_{p}} \frac{v_{g}}{c} \quad$ and $\xi=\frac{\omega_{p}}{c} z+U_{g} \tau$, Eq. (26) can be written as:

$i \frac{\partial a}{\partial \tau}+\frac{1}{2} \frac{\partial^{2} a}{\partial \zeta^{2}}+D_{\mathrm{NL}} a=0$,

where $D_{\mathrm{NL}}=N|a|^{2} / 2$. Equation (27) is the well-known nonlinear Schrödinger equation (NLSE). This equation is frequently met in different areas of theoretical physics, especially in nonlinear optics. The NLSE describes the propagation of waves in nonlinear media taking into account both the group velocity dispersion (second term) and the nonlinearity (third term). It is a classical field equation whose important applications are in the propagation of EMWs in nonlinear optical fibers and planar waveguides [59] and to Bose-Einstein condensates confined to highly anisotropic cigar-shaped traps, in the meanfield regime [60]. Additionally, it can be revealed in the studies of small-amplitude gravity waves on the surface of deep zero-viscosity water [59], Langmuir waves in hot plasmas [59], propagation of plane-diffracted wave beams in the focusing areas of the ionosphere [61] and propagation of Davydov's alpha-helix solitons, which are responsible for energy transport along molecular chains [62].

The MI for the right- and left-hand circularly polarized EMW can be obtained using the usual method introduced by Shukla et al. [46]. In this approach, we suppose:

$a=\left(a_{0}+a_{1}\right) \exp (i \Lambda \tau)$,

where $a_{0}$ is a real constant and $a_{0}>>\left|a_{1}\right|$,

$\Lambda \equiv D_{\mathrm{NL}}\left(a=a_{0}\right)=\frac{1}{2} a_{0}^{2} N$.
By substituting Eq. (28) into Eq. (27) and linearizing obtained equation with respect to $a_{1}$, we can achieve:

$i \frac{\partial a_{1}}{\partial \tau}+\frac{1}{2} \frac{\partial^{2} a_{1}}{\partial \zeta^{2}}+\frac{1}{2} a_{0}^{2} N\left(a_{1}+a_{1}^{*}\right)=0$.

Introducing $a_{1}=X+i Y$, inserting it into Eq. (30) and separating the real and imaginary parts of this equation yield:

$\left\{\begin{aligned} \frac{\partial X}{\partial \tau}+\frac{1}{2} \frac{\partial^{2} Y}{\partial \zeta^{2}} & =0 \\ -\frac{\partial Y}{\partial \tau}+\frac{1}{2} \frac{\partial^{2} X}{\partial \zeta^{2}}+a_{0}^{2} N & =0\end{aligned}\right.$

We consider the following oscillational form for $X$ and $Y$ :

$\left(\begin{array}{c}X \\ Y\end{array}\right)=\left(\begin{array}{c}\tilde{X} \\ \tilde{Y}\end{array}\right) \exp (-i \Omega \tau+i K \zeta)$

where $\tilde{X}$ and $\tilde{Y}$ are real amplitudes, $\Omega$ is the modulation frequency normalized by $\omega_{p}^{2} / \omega_{0}$ and $K$ is the modulation wave number normalized by $\omega_{p} / c$. By substituting Eq. (32) into the set of Eq. (31), we can obtain the following nonlinear dispersion relation of $\mathrm{MI}$ :

$\Omega^{2}=-\frac{K^{2}}{2}\left[a_{0}^{2} N-\frac{K^{2}}{2}\right]$.

The temporal growth rate $\Gamma=-i \Omega$ can be extracted from Eq. (33) as below:

$\Gamma=\frac{K}{\sqrt{2}}\left[a_{0}^{2} N-\frac{K^{2}}{2}\right]^{1 / 2}$.

The maximum growth rate of $\mathrm{MI}$ that occurs at $K=$ $K_{m}=a_{0} N^{1 / 2}$ is

$\Gamma_{\max }=\frac{a_{0}^{2}}{2} N$.

It may be useful to note that for e-i plasma, when $\zeta_{i}=1$ and $\zeta_{\mathrm{d}+}=\zeta_{\mathrm{d}-}=0$, Eq. (33) reduces to,

$\Gamma=\frac{K}{\sqrt{2}}\left[\frac{a_{0}^{2}}{2(1-\sigma \alpha)^{2}}\left(\frac{\beta_{e}}{\left(1+T_{e} / T_{i}\right)}+\frac{1}{(1-\sigma \alpha)^{2}}\right)-\frac{K^{2}}{2}\right]^{1 / 2}$.

\section{Numerical discussions}

For numerical studies, in all the investigated cases, we suppose an Nd:YAG laser with frequency $\omega_{0}=1.88 \times 10^{15} \mathrm{~s}^{-1}$ (that corresponds to the laser wave length $\lambda \approx 1 \mu \mathrm{m}$ ) and $a_{0}=0.271$ (laser intensity $I \approx 10^{17} \mathrm{~W} / \mathrm{cm}^{2}$ ); also, we 


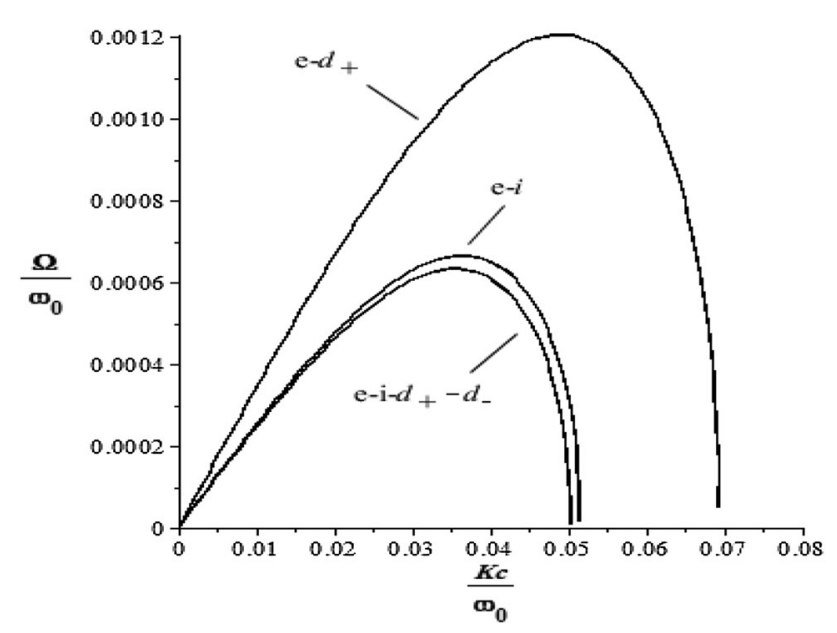

Fig. 1 Variations of the normalized MI growth rate with respect to the normalized modulation frequency for three different cases, e-i plasma with $\eta=\xi=0$, e-d + plasma with $\eta=10^{-1}, \xi=0$ and e-i$\mathrm{d}+-\mathrm{d}-$ plasma with $\eta=\xi=5 \times 10^{-2}$, when $z_{+}=z_{-}=10$

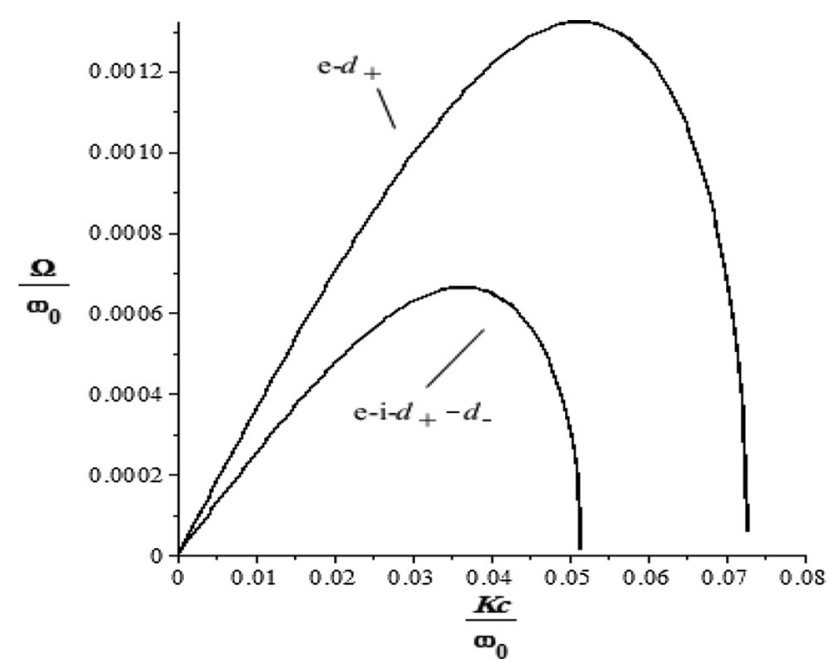

Fig. 2 Variations of the normalized MI growth rate with respect to the normalized modulation frequency for two different cases, e-d+ plasma with $\eta=10^{-3}, \xi=0$ and $\mathrm{e}-\mathrm{i}-\mathrm{d}+-\mathrm{d}-$ plasma with $\eta=\xi=5 \times 10^{-4}$, when $z_{+}=z_{-}=1000$

consider only the right-hand polarization laser in magnetized medium with $\alpha=0.2$ and fix the temperature $T_{j}=1 \mathrm{keV}$ for all the plasma components. For more clarification, we introduce two new parameters $\eta$ and $\xi$ as:

$n_{0 \mathrm{~d}+}=\eta n_{0}, \quad n_{0 \mathrm{~d}_{-}}=\xi n_{0}$,

where we supposed $n_{0 i}+z_{+} n_{0 \mathrm{~d}+}=n_{0 e}+z_{-} n_{0 \mathrm{~d}-}=n_{0}$ and set $n_{0}=10^{17} \mathrm{~cm}^{-3}$.

Figure 1 shows variations of the normalized modulation growth rate $\Omega / \omega_{0}$ with respect to the normalized modulation wave number $K c / \omega_{0}$, when $z_{+}=z_{-}=10$. We have three different cases, in which $\eta=\xi=0$ corresponds to the e- $\mathrm{i}$ plasma, $\eta=10^{-1}, \xi=0$ to the e-d+plasma and $\eta=\xi=$ $5 \times 10^{-2}$ to the e-i-d+-d-plasma. We can see that adding positive dust grains instead of ions substantially increases the modulation growth rate, because localization of positive charges on the dust grains improves the ambipolar potential, which in turn leads to the sharpness of the density profile and consequently to more modulation. In the case of $\mathrm{e}-\mathrm{i}-\mathrm{d}+-\mathrm{d}-$ plasma, decreasing the density of electrons and adding equivalent negative dust grains to the plasma results in the decrease in the laser modulation growth rate, because the nonlinear current is created by the motion of the electrons and decrease in the population of electrons leads to the decrease in the nonlinearity of the medium and consequently to the decrease in the growth rate. To investigate the effect of the order of ionization of dust grains on the spot size, in Fig. 2, we choose $z_{+}=z_{-}=1000$ for two different cases, the e-d + plasma with $\eta=10^{-3}, \xi=0$ and the e-i-d+-dplasma with $\eta=\xi=5 \times 10^{-5}$. We can see that the increase in the ionization order causes a small increase in the MI growth rate. It is worth mentioning that our numerical experiments show that an increase in the temperature causes a decrease in the modulation growth rate. In addition, magnetization of plasma enhances the modulation growth rate for the right-hand polarization and inversely reduces it for the lefthand one. These results are not new and have been investigated earlier [53, 54]; for brevity we do not provide them.

\section{Conclusions}

In this paper, we investigated the MI of a weakly relativistic laser propagating along an external magnetic field in the hot plasma containing positive and negative dust grains. The MI growth rate of the circularly polarized laser beam in the dusty plasma was obtained. It was found that adding the positive dust grains to plasma enhances the MI, but existence of the negative dust grains reduces it. Furthermore, the effect of the order of dust grain ionization on the MI was investigated and it was observed that its increase leads to the increase in the MI growth rate.

Open Access This article is distributed under the terms of the Creative Commons Attribution 4.0 International License (http://crea tivecommons.org/licenses/by/4.0/), which permits unrestricted use, distribution, and reproduction in any medium, provided you give appropriate credit to the original author(s) and the source, provide a link to the Creative Commons license, and indicate if changes were made.

\section{References}

1. Goertz, C.K.: Dusty plasmas in the solar system. Rev. Geophys. 27, 271 (1989) 
2. Northrop, T.G.: Dusty plasmas. Phys. Scr. 45, 475 (1992)

3. Tsytovich, V.N.: Dust plasma crystals, drops, and clouds. Usp. Fiz. Nauk 167, 57 (1997). [Phys. Usp. 40, 53 (1997)]

4. Bliokh, P., Sinitsin, V., Yaroshenko, V.: Dusty and self-gravitational plasmas in space. Kluwer Acad. Publ, Dordrecht (1995)

5. Shukla, P.K., Mamun, A.A.: Introduction to dusty plasma physics. Institute of Physics Publishing, Bristol (2002)

6. Whipple, E.C.: Potentials of surfaces in space. Rep. Prog. Phys. 44, 1197 (1981)

7. Robinson, P.A., Coakley, P.: Spacecraft charging-progress in the study of dielectrics and plasmas. IEEE Trans. Electr. Insul. 27, 944 (1992)

8. Tsytovich, V.N., Winter, J.: On the role of dust in fusion devices. Usp. Fiz. Nauk 168, 899 (1998). [Phys. Usp. 41, 815 (1998)]

9. Winter, J., Gebauer, G.: Dust in magnetic confinement fusion devices and its impact on plasma operation. J. Nucl. Mater. 266-269, 228 (1999)

10. Winter, J.: Dust: a new challenge in nuclear fusion research? Phys. Plasmas 7, 3862 (2000)

11. Jellum, G.M., Graves, D.B.: Particulates in aluminum sputtering discharges. J. Appl. Phys. 67, 6490 (1990)

12. Anderson, H.M., Jairath, R., Mock, J.L.: Particulate generation in silane/ammonia rf discharges. J. Appl. Phys. 67, 3999 (1990)

13. Shivatani, M., Fukuzawa, T., Watanabe, Y.: Formation processes of particulates in helium-diluted silane RF plasmas. IEEE Trans. Plasma Sci. 22, 103 (1994)

14. Cui, C., Goree, J.: Fluctuations of the charge on a dust grain in a plasma. IEEE Trans. Plasma Sci. 22, 151 (1994)

15. Trajanovic, Z., Senapati, L., Sharma, R.P., Venkatesan, T.: Stoichiometry and thickness variation of $\mathrm{YBa}_{2} \mathrm{Cu}_{3} \mathrm{O}_{7-x}$ in off-axis pulsed laser deposition. Appl. Phys. Lett. 66, 2418 (1995)

16. Fukushima, K., Kanka, Y., Badaye, M., Morishita, T.: Velocity distributions of ions in the ablation plume of a $\mathrm{Y}_{1} \mathrm{Ba}_{2} \mathrm{Cu}_{3} \mathrm{O}_{x}$ target. J. Appl. Phys. 77, 5406 (1995)

17. Abrahamson, J., Dinniss, J.: Ball lightning caused by oxidation of nanoparticle networks from normal lightning strikes on soil. Nature 403, 519 (2000)

18. Jana, M.R., Sen, A., Kaw, P.K.: Collective effects due to chargefluctuation dynamics in a dusty plasma. Phys. Rev. E 48, 3930 (1993)

19. Sambandan, G., Tripathi, V.K., Parashar, J., Bharuthram, R.: Nonlinear interaction of a high-power electromagnetic beam in a dusty plasma: two-dimensional effects. Phys. Plasmas 6, 762 (1999)

20. Sharma, S.C., Gahlot, A., Sharma, R.P.: Effect of dust on an amplitude modulated electromagnetic beam in a plasma. Phys. Plasmas 15, 043701 (2008)

21. Sodha, M.S., Mishra, S.K., Misra, S.: Nonlinear dependence of complex plasma parameters on applied electric field. Phys. Plasmas 18, 023701 (2011)

22. El-Taibany, W.F., Kourakis, I., Wadati, M.: Low frequency localized wavepackets in dusty plasmas with opposite charge polarity dust components. Plasma Phys. Control. Fusion 50, 074003 (2008)

23. Varma, R.K., Shukla, P.K., Krishan, V.: Electrostatic oscillations in the presence of grain-charge perturbations in dusty plasmas. Phys. Rev. E 47, 3612 (1993)

24. Tripathi, K.D., Sharma, S.K.: Self-consistent charge dynamics in magnetized dusty plasmas: low-frequency electrostatic modes. Phys. Rev. E 53, 1035 (1996)

25. Das, C., Janaki, M.S., Dasgupta, B.: Dust cyclotron instability in presence of dust charge fluctuations. Phys. Scr. T 75, 216 (1998)

26. Dwivedi, C.B., Pandey, B.P.: Electrostatic shock wave in dusty plasmas. Phys. Plasmas 2, 4134 (1995)

27. Popel, S.I., Yu, M.Y., Tsytovich, V.N.: Shock waves in plasmas containing variable-charge impurities. Phys. Plasmas 3, 4313 (1996)
28. Paul, S.N., Mondal, K.K., Roychowdhury, A.: Effects of streaming and attachment coefficients of ions and electrons on the formation of soliton in a dusty plasma. Phys. Lett. A 257, 165 (1999)

29. Janaki, M.S., Dasgupta, B.: Surface waves in a dusty plasma. Phys. Scr. 58, 493 (1998)

30. Alam, M.K., Roy Chowdhury, A.: Surface wave propagation in a magnetized dusty plasma with charge fluctuation. Phys. Plasmas 6, 3765 (1999)

31. Bhat, J.R., Pandey, B.P.: Self-consistent charge dynamics and collective modes in a dusty plasma. Phys. Rev. E 50, 3980 (1994)

32. Burman, S., Paul, S.N., Roy Chowdhury, A.: Stimulated brillouin scattering in a magnetized dusty plasma with charge fluctuation. Phys. Plasmas 9, 3752 (2002)

33. Mishra, S.K., Mishra, S., Sodha, M.S.: Self-focusing of a Gaussian electromagnetic beam in a complex plasma. Phys. Plasmas 18, 043702 (2011)

34. Nantel, M., Ma, G., Gu, S., Cote, C.Y., Itatani, J., Umstadter, D.: Pressure ionization and line merging in strongly coupled plasmas produced by 100-fs laser pulses. Phys. Rev. Lett. 80, 4442 (1998)

35. Springate, E., Hay, N., Tisch, J.W.G., Mason, M.B., Ditmire, T., Hutchinson, M.H.R., Marangos, J.P.: Explosion of atomic clusters irradiated by high-intensity laser pulses: scaling of ion energies with cluster and laser parameters. Phys. Rev. A 61, 063201 (2000)

36. Last, I., Jortnera, J.: Electron and nuclear dynamics of molecular clusters in ultraintense laser fields. I. Extreme multielectron ionization. J. Chem. Phys. 120, 1336 (2004)

37. Stoffels, E., Stoffels, W.W., Vender, D., Kroesen, G.M.W., de Hoog, F.J.: Laser-particulate interactions in a dusty RF plasma. IEEE Trans. Plasma Sci. 22, 116 (1994)

38. Melzer, A.: Laser-experiments on particle interactions in strongly coupled dusty plasma crystals. Phys. Scr. T89, 33 (2001)

39. Nefedov, A.P., Petrov, O.F., Molotkov, V.I., Fortovs, V.E.: Formation of liquidlike and crystalline structures in dusty plasmas. JETP Lett. 72, 218 (2000)

40. van de Wetering, F.M.J.H., Oosterbeek, W., Beckers, J., Nijdam, S., Gibert, T., Mikikian, M., Rabat, H., Kovačević, E., Berndt, J.: Interaction of nanosecond ultraviolet laser pulses with reactive dusty plasma. Appl. Phys. Lett. 108, 213103 (2016)

41. Nosenko, V., Avinash, K., Goree, J., Liu, B.: Laser-excited mach cones in a dusty plasma crystal. Phys. Rev. E 62, 4162 (2000)

42. van de Wetering, F.M.J.H., Oosterbeek, W., Beckers, J., Nijdam, S., Kovačević, E., Berndt, J.: Laser-induced incandescence applied to dusty plasmas. J. Phys. D Appl. Phys. 49, 295206 (2016)

43. Esarey, E., Sprangle, P., Krall, J., Ting, A.: Overview of plasmabased accelerator concepts. IEEE Trans. Plasma Sci. 24, 252 (1996)

44. Esarey, E., Sprangle, P., Krall, J., Ting, A.: Self-focusing and guiding of short laser pulses in ionizing gases and plasmas. IEEE J. Quantum Electron. 33, 1879 (1997)

45. Shukla, P.K., Marklund, M., Eliasson, B.: Nonlinear dynamics of intense laser pulses in a pair plasma. Phys. Lett. A 324, 193 (2004)

46. Shukla, P.K., Bharuthram, R.: Modulational instability of strong electromagnetic waves in plasmas. Phys. Rev. A 35, 4889 (1987)

47. McKinstrie, C.J., Bingham, R.: Stimulated Raman forward scattering and the relativistic modulational instability of light waves in rarefied plasma. Phys. Fluids B 4, 2626 (1992)

48. Sprangle, P., Esarey, E., Hafizi, B.: Intense laser pulse propagation and stability in partially stripped plasmas. Phys. Rev. Lett. 79, 1046 (1997)

49. Shukla, P.K., Rao, N.N., Yu, M.Y., Tsintsadze, N.L.: Relativistic nonlinear effects in plasmas. Phys. Rep. 138, 1 (1986)

50. Esarey, E., Kralland, J., Sprangle, P.: Envelope analysis of intense laser pulse self-modulation in plasmas. Phys. Rev. Lett. 72, 2887 (1994) 
51. Jha, P., Kumar, P., Raj, G., Upadhyaya, A.K.: Modulation instability of laser pulse in magnetized plasma. Phys. Plasmas 12, 123104 (2005)

52. Chen, H.Y., Liu, S.Q., Li, X.Q.: Self-modulation instability of an intense laser beam in a magnetized pair plasma. Phys. Scr. 83, 035502 (2011)

53. Sepehri Javan, N.: Modulation instability of an intense laser beam in the hot magnetized electron-positron plasma in the quasineutral limit. Phys. Plasmas 19, 122107 (2012)

54. Sepehri Javan, N.: Competition of circularly polarized laser modes in the modulation instability of hot magnetoplasma. Phys. Plasmas 20, 012120 (2013)

55. Etemadpour, R., Sepehri, N.: Javan, Effect of super-thermal ions and electrons on the modulation instability of a circularly polarized laser pulse in magnetized plasma. Laser Part. Beams 33, 265 (2015)

56. Sepehri Javan, N., Nasirzadeh, Zh: Self-focusing of circularly polarized laser pulse in the hot magnetized plasma in the quasineutral limit. Phys. Plasmas 19, 112304 (2012)
57. Sepehri Javan, N., Rouhi Erdi, F.: Effect of dynamical nonneutrality on the modulational instability of laser propagating through hot magnetoplasma. Phys. Plasmas 22, 062116 (2015)

58. Sepehri Javan, N., Hosseinpour Azad, M.: Thermal behavior change in the self-focusing of an intense laser beam in magnetized electron-ion-positron plasma. Beams 32, 321 (2014)

59. Malomed, B.: Nonlinear Schrödinger equations. In: Scott, A. (ed.) Encyclopedia of nonlinear science, pp. 639-643. Routledge, New York (2005)

60. Pitaevskii, L., Stringari, S.: Bose-Einstein condensation. Clarendon, Oxford (2003)

61. Gurevich, A.V.: Nonlinear phenomena in the ionosphere. Springer, Berlin (1978)

62. Balakrishnan, R.: Soliton propagation in nonuniform media. Phys. Rev. A. 32, 1144 (1985) 